\title{
Does cachexia prevention improve outcome of chronic disease and cancer?
}

\author{
Gianni Biolo $\cdot$ Martina Guadagni $\cdot$ Beniamino Ciocchi
}

Received: 30 December 2010/Accepted: 21 January 2011/Published online: 24 February 2011

(C) SIMI 2011

\section{Introduction}

One of the greatest achievements in medicine is the potential of new therapies to stabilize chronic illness and cancer. In many cases, the disease cannot be cured completely, but is effectively controlled for many years. These medical advancements have substantially contributed to decrease age-related disability, and to increase active-life expectancy of the population. In addition to specific therapies, patients with cancer and chronic diseases will require integrated health care programs to maintain functional autonomy and quality of life. These adjuvant interventions are mainly based on nutritional support and exercise programs. Nonetheless, malnutrition is commonly seen in patients with cancer or chronic diseases, such as heart failure, coronary artery disease, chronic obstructive pulmonary disease (COPD), hepatic cirrhosis, rheumatoid arthritis, end-stage kidney disease, human immunodeficiency virus (HIV) infection, diabetes, etc. Long-term activation of the systemic inflammatory reaction combined with poor nutrition and physical inactivity are the key mechanisms leading to cachexia. This clinical syndrome includes severe muscle wasting, fatigue, hypoalbuminemia,

Commentary to the article "The parallel pathway: a novel nutritional and metabolic approach to cancer patients". by Muscaritoli et al., Intern Emerg Med (Epub ahead of print) July 2.

G. Biolo · M. Guadagni · B. Ciocchi

Division of Internal Medicine, Department of Medical,

Technological and Translational Sciences,

AOUTS, University of Trieste, Trieste, Italy

G. Biolo $(\bowtie)$

Clinica Medica, Ospedale di Cattinara,

Strada di Fiume 447, 34149 Trieste, Italy

e-mail: biolo@units.it impaired immune response, anemia, anorexia and loss of fat. Late-stage cachexia is substantially untreatable, and negatively affects the quality of life of patients as well as interfering with therapy ultimately being a primary cause of morbidity and mortality. At earlier stages, malnutrition can be reversed by appropriate nutritional interventions. Diagnostic criteria for pre-cachexia have recently been defined in order to identify patients at risk of malnutrition [1].

In this issue of Internal and Emergency Medicine, Muscaritoli et al. [2] describe a multi professional and multimodal approach aimed at preventing or delaying cancer-related malnutrition. They suggest that monitoring of nutritional and metabolic abnormalities as well as metabolic care should start at early stages of the disease, and proceed in parallel with the specific oncological therapies. Metabolic intervention should include nutritional counselling, supplement prescription or artificial nutrition (enteral or parenteral) as well as other support interventions such as exercise and physiotherapy. This approach, defined as a "parallel pathway, was primarily aimed at improving the efficacy of oncological therapies and surgery, and ultimately, at improving patient clinical outcome, and possibly, survival. In fact, it is well established that malnutrition reduces the effectiveness of therapies used for cancer treatment, while chemotherapy and radiotherapy can also negatively influence the nutritional status of patients. Randomized controlled trials (RCTs) indicate that interventions based on enteral or parenteral nutrient supplementations, when prescribed in parallel with chemoradiotherapy or surgery, may improve quality of life and reduce therapy related side-effects and mortality.[3-6].

RCTs also suggest that improving nutritional status is not only beneficial in cancer, but also in patients affected by different chronic diseases. Nutritional support in COPD 
patients improves muscle strength and pulmonary function as well as decreases the frequency of acute exacerbations $[7,8]$. Enteral nutrition is effective in improving the clinical outcome in severely malnourished patients with hepatic cirrhosis [9]. Nutritional interventions increase serum levels of albumin in dialysis patients [10], and improve the clinical status and quality of life in chronic heart failure patients [11]. In developing countries, food assistance programs targeted to HIV-infected individuals delay the disease progression [12]. Among contacts of tuberculosis cases, malnutrition is identified as the main risk-factor for the development of the disease [13].Finally, meta-analyses indicate that enteral nutritional support can significantly reduce the risk of developing pressure ulcers [14], and may decrease mortality in older people with chronic diseases who are undernourished [15].

This evidence clearly shows that nutritional interventions to prevent disease-associated malnutrition have the potential to modify the course of chronic diseases, and to improve patient outcome. However, the application of an evidence-based approach to clinical nutrition has always not been successful. RCTs are universally regarded as the gold standard by which, to determine whether a specific treatment is appropriate in a particular clinical situation. However, RCTs may not be particularly appropriate in the evaluation of the effects of nutritional interventions in chronic diseases. There are three major factors limiting the ability of RCTs to detect the effects of nutritional interventions on outcomes. First, the effect of an adjuvant intervention, as nutrition, is usually smaller in scale than the primary therapy for the disease. Nutritional studies, therefore, require large number of participants to be adequately powered. Second, nutritional interventions require a long-time exposure to modify clinical outcomes particularly in chronic diseases. Third, malnourished patients are usually excluded from nutritional RCTs, because withholding specialized nutritional support from them is considered unethical. As a consequence of these limitations, an insufficient number of "high-quality" RCTs have been published to date in the field of clinical nutrition to support a real evidence-based nutrition. Nonetheless, despite the fact that clear cut evidence is often lacking in some areas of clinical nutrition, guidelines unanimously indicate that cachexia prevention should be among the primary goals in cancer and chronic disease treatments. Descriptive epidemiological studies showing strict associations between cachexia and disease progression are often regarded as sufficient evidence to justify nutritional recommendations. We agree with Muscaritoli et al. [2], therefore, that the "parallel pathway", as described for cancer patients, could be also implemented into the therapeutical strategies of other chronic diseases, particularly in COPD, acquired immune deficiency syndrome, hepatic cirrhosis, end-stage kidney disease and heart failure. Patients who are candidates for organ transplantation would particularlybenefit from early detection and treatment of malnutrition.

Finally, the "parallel pathway" has been specifically designed to improve the efficacy of specific disease therapies through nutritional support. This approach must be distinguished from nutrition in palliative care of patients whose conditions do not respond to curative treatment. The emphasis of palliative nutrition is on a quality of life and symptom relief rather than active nutritional therapy. In these patients, nutritional intervention should be considered and assessed individually.

\section{Conflict of interest None.}

\section{References}

1. Muscaritoli M, Anker SD, Argilés J, Aversa Z, Bauer JM, Biolo G, Boirie Y, Bosaeus I, Cederholm T, Costelli P, Fearon KC, Laviano A, Maggio M, Rossi Fanelli F, Schneider SM, Schols A, Sieber CC (2010) Consensus definition of sarcopenia, cachexia and pre-cachexia: joint document elaborated by Special Interest Groups (SIG) "cachexia-anorexia in chronic wasting diseases" and "nutrition in geriatrics". Clin Nutr 29:154-159

2. Muscaritoli M, Molfino A, Gioia G, Laviano A, Rossi Fanelli F (2010) The "parallel pathway": a novel nutritional and metabolic approach to cancer patients. Intern Emerg Med. doi:10.1007/ s11739-010-0426-1

3. Ravasco P, Monteiro-Grillo I, Marques Vidal P, Camilo ME (2005) Impact of nutrition on outcome: a prospective randomized controlled trial in patients with head and neck cancer undergoing radiotherapy. Head Neck 27:659-668

4. Hasenberg T, Essenbreis M, Herold A, Post S, Shang E (2010) Early supplementation of parenteral nutrition is capable of improving quality of life, chemotherapy-related toxicity and body composition in patients with advanced colorectal carcinoma undergoing palliative treatment. Results from a prospective, randomized clinical trial. Colorectal Dis 12:190-199

5. Paccagnella A, Morello M, Da Mosto MC, Baruffi C, Marcon ML, Gava A, Baggio V, Lamon S, Babare R, Rosti G, Giometto M, Boscolo-Rizzo P, Kiwanuka E, Tessarin M, Caregaro L, Marchiori C (2010) Early nutritional intervention improves treatment tolerance and outcomes in head and neck cancer patients undergoing concurrent chemoradiotherapy. Support Care Cancer 18:837-845

6. Buijs N, van der Schueren MA, Langius JA, Leemans CR, Kuik DJ, Vermeulen MA, van Leeuwen PA (2010) Perioperative arginine-supplemented nutrition in malnourished patients with head and neck cancer improves long-term survival. Am J Clin Nutr 92:1151-1156

7. Creutzberg EC, Wouters EF, Mostert R, Weling-Scheepers CA, Schols AM (2003) Efficacy of nutritional supplementation therapy in depleted patients with chronic obstructive pulmonary disease. Nutrition 19:120-127

8. Weekes CE, Emery PW, Elia M (2009) Dietary counselling and food fortification in stable COPD: a randomised trial. Thorax 64:326-331

9. Cabre E, Gonzalez-Huix F, Abad-Lacruz A, Esteve M, Acero D, Fernandez-Bañares F, Xiol X, Gassull MA (1990) Effects of total enteral nutrition on the short-term outcome of severely malnourished cirrhotics. A randomized controlled trial. Gastroenterology 98:715-720 
10. Moretti HD, Johnson AM, Keeling-Hathaway TJ (2009) Effects of protein supplementation in chronic hemodialysis and peritoneal dialysis patients. J Ren Nutr 19:298-303

11. ColínRamírez E, Castillo Martínez L, Orea Tejeda A, Rebollar González V, Narváez David R, Asensio Lafuente E (2004) Effects of a nutritional intervention on body composition, clinical status, and quality of life in patients with heart failure. Nutrition 20:890-895

12. Rawat R, Kadiyala S, McNamara PE (2010) The impact of food assistance on weight gain and disease progression among HIVinfected individuals accessing AIDS care and treatment services in Uganda. BMC Public Health 10:316
13. Morán-Mendoza O, Marion SA, Elwood K, Patrick D, FitzGerald JM (2010) Risk factors for developing tuberculosis: a 12-year follow-up of contacts of tuberculosis cases. Int J Tuberc Lung Dis 14:1112-1119

14. Stratton RJ, Ek AC, Engfer M, Moore Z, Rigby P, Wolfe R, Elia M (2005) Enteral nutritional support in prevention and treatment of pressure ulcers: a systematic review and meta-analysis. Ageing Res Rev 4:422-450

15. Milne AC, Potter J, Vivanti A, Avenell A (2009) Protein and energy supplementation in elderly people at risk from malnutrition. Cochrane Database Syst Rev 2:CD003288 